\title{
THE FOURIER MULTIPLIER PROBLEM FOR SPACES OF CONTINUOUS FUNCTIONS WITH $p$-SUMMABLE TRANSFORMS
}

Dedicated to the memory of Hanna Neumann

LYNETTE M. BLOOM

(Received 29 June 1972)

Communicated by M. F. Newman

\section{Introduction}

In this paper we consider spaces $A^{p}, p \in[1,2]$, and multipliers $\left(A^{p}, A^{q}\right)$, $p \in[1,2], q \in[1,2]$. In 4.4 and 6.1 we identify $\left(A^{p}, A^{q}\right)$ for $p \in[1,2], q \in[p, 2]$, and in 7.3 we identify $\left(A^{2}, A^{1}\right)$. In 7.1 we give a sufficient condition, and in 7.5 a necessary condition, for membership of $\left(A^{p}, A^{q}\right), p \in(1,2), q \in[1, p)$. We give, in 7.2, a necessary condition for membership of $\left(A^{2}, A^{q}\right), q \in[1,2)$. We include constructive proofs of some strict inclusion results for $A^{p}, p \in[1,2],(3.1$ and 3.2), and also, in 5.3, for $\left(A^{p}, A^{p}\right), p \in[1,2]$.

The author would like to thank Professor Robert Edwards for his many helpful suggestions and for his constant guidance during the work for this paper.

\section{Preliminaries}

2.1 We consider functions on the circle group $\boldsymbol{T}$, and write

$$
A^{p}=\left\{f \in C(T): \hat{f} \in l^{p}(Z)\right\}, \quad p \in[1, \infty) ;
$$

compare here the author's paper [1]. It is known that $A^{p}$ is a Banach space under the norm

$$
N_{p}: h \mapsto\|h\|_{\infty}+\|\hat{h}\|_{p}=\|h\|_{\infty}+M_{p}(h) .
$$

We define $e_{v}$ to be the function $e^{i t} \leftrightarrow e^{i v t}$ on $T$ and note that, for $h \in A^{p}$,

$$
N_{p}\left(e_{v} h\right)=N_{p}(h) ; M_{p}\left(e_{v} h\right)=M_{p}(h) .
$$

The spectrum of $h \in L^{1}(T)$ is defined by

$$
\operatorname{sp}(h)=\{n \in Z: \hat{h}(n) \neq 0\} .
$$

If $\phi, \psi$ are positive functions on $\{0,1,2, \cdots\}$, we write $\phi \sim \psi$ if and only if $0<\inf \phi^{-1} \psi \leqq \sup \phi^{-1} \psi<\infty$. 
2.2 In [4], p. 33, the Rudin-Shapiro polynomials $P_{m}(m=0,1,2, \cdots)$ are defined by

$$
P_{m}=\sum_{n=0}^{2 m-1} \varepsilon_{m}(n) e_{n},
$$

where the $\varepsilon_{m}(n) \in\{-1,1\}$ are chosen in such a way that

$$
\left|P_{m}\right| \leqq 2^{(m+1) / 2} ; M_{p}\left(P_{m}\right)=2^{m / p}, \quad m=0,1,2, \cdots .
$$

2.3 By a multiplier from $A^{p}$ to $A^{q}, p \in[1,2], q \in[1,2]$, we mean a continuous linear operator $T: A^{p} \rightarrow A^{q}$ which commutes with translations. As can be seen from [2], 16.3.1, to each multiplier $T: A^{p} \rightarrow A^{q}$ there corresponds a unique distribution $\phi$ such that $T$ is (the restriction to $A^{p}$ of) the operator $T_{\phi}$ defined by

$$
T_{\phi} f=\phi * f .
$$

We denote the space of such distributions $\phi$ by $\left(A^{p}, A^{q}\right)$ and refer to $\phi \in\left(A^{p}, A^{q}\right)$ as a multiplier from $A^{p}$ to $A^{q}$. A distribution $\phi$ belongs to $\left(A^{p}, A^{q}\right)$ if and only if

$$
N_{q}(\phi * f) \leqq \text { const. } N_{p}(f), \forall f \in T P,
$$

where $T P$ denotes the space of trigonometric polynomials on $T$. In particular, a distribution $\phi$ belongs to $\left(A^{p}, C\right)=\left(A^{p}, A^{2}\right)$ if and only if

$$
\|\phi * f\|_{\infty} \leqq \text { const. } N_{p}(f), \forall f \in T P ;
$$

or, what is equivalent, if and only if

$$
|\phi * f(1)| \leqq \text { const. } N_{p}(f), \forall f \in T P .
$$

2.4 We denote by $P M$ the space of pseudomeasures on $T$, and those pseudomeasures having Fourier transforms in $l^{k}, k \in(0, \infty]$, we denote by $P M^{k} . P M^{1}$ is identifiable with $A=A^{1}, P M^{2}$ with $L^{2}$, and $P M^{\infty}$ with $P M$. We denote by $M$ the space of Radon measures on $T$, and by $M^{k}$ those measures having Fourier transforms in $l^{k}, k \in(0, \infty] . M^{2}$ is identifiable with $L^{2}$.

2.5 We write $p^{\prime}$ for the conjugate exponent of $p \in[1, \infty)$. $p^{\prime}$ is such that $1 / p+1 / p^{\prime}=1, p \in(1, \infty)$, and $p^{\prime}=\infty$ if $p=1$.

2.6 We define $\left(A^{p}\right)^{\prime}$ to be the set of linear functionals $l$ on $T P$ such that

$$
|l(f)| \leqq \text { const. } N_{p}(f), \quad \forall f \in T P .
$$

Since $T P$ is dense in $A^{p}$, the restriction from $A^{p}$ to $T P$ gives a $1-1$ map of the dual of $A^{p}$ onto $\left(A^{p}\right)^{\prime}$.

2.7 For $a \in T$, we define translation operators $\tau_{a}$ by

$$
\tau_{a} f: x \mapsto f(a x), \forall x \in T .
$$




\section{Strict inclusion results for $A^{p}, p \in[1,2]$}

In this section we will prove constructively the following strict inclusions:

and

$$
\bigcup_{p \in[1, q)} A^{p} \underset{\neq}{\subsetneq} A^{q} \text { if } q \in(1,2],
$$

$$
A^{q} \underset{p}{\varsubsetneqq} \bigcap_{p \in(q .2]} A^{p} \text { if } q \in[1,2) .
$$

Construction 3.1 The strict inclusion (3.1).

Consider a given $q \in(1,2]$. Define $f_{k} \in T P$ by

$$
f_{k}=\beta_{k, q} P_{k} e_{v_{k}}, \quad k=0,1,2, \cdots,
$$

where the sequences $\left(\beta_{k, q}\right)$ and $\left(v_{k}\right)$ will be chosen appropriately, the latter in such a way to ensure that the $S_{k}=\operatorname{sp}\left(f_{k}\right)$ are disjoint. Now, from (2.1), (2.2) and (3.3) we have

$$
N_{q}\left(f_{k}\right) \sim \beta_{k, q} 2^{k / q}, q \in(1,2],
$$

and

$$
N_{p}\left(f_{k}\right) \sim \beta_{k, q} 2^{k / p}, p \in[1, q) .
$$

Define $f=\sum_{k=0}^{\infty} f_{k}$. Since $N_{q}(f) \leqq \sum_{k=0}^{\infty} N_{q}\left(f_{k}\right)$ it follows from (3.4) that a sufficient condition for $f \in A^{q}$ is that

$$
\sum_{k=0}^{\infty} \beta_{k . q} 2^{k / q}<\infty, q \in(1,2]
$$

Choose

$$
\beta_{k . q}=(k+1)^{-2} 2^{-k / q}, \quad k=0,1,2, \cdots .
$$

Then (3.6) is satisfied since

$$
\sum_{k=0}^{\infty} \beta_{k, q} 2^{k / q}=\sum_{k=0}^{\infty}(k+1)^{-2}<\infty .
$$

We will now show that, with $\left(\beta_{k . q}\right)$ as in (3.7), $f \notin \bigcup_{p \in[1, q)} A^{p}$. Since the series defining $f$ converges in $A^{q}$,

$$
f(n)= \begin{cases}\hat{f}_{k}(n) & n \in S_{k}, \quad k=0,1,2, \cdots, \\ 0 & n \notin \bigcup_{k} S_{k},\end{cases}
$$

where

$$
\hat{f}_{k}(n)= \begin{cases}\varepsilon_{k}(n)(k+1)^{-2} 2^{-k / q} & n \in S_{k} \\ 0 & n \notin S_{k}\end{cases}
$$


Also, for $f_{k}$ defined as in (3.3),

$$
S_{k}=\left\{n \in Z: v_{k} \leqq n \leqq v_{k}+2^{k}-1\right\},
$$

so each $S_{k}$ is a finite set with cardinality

$$
\left|S_{k}\right|=2^{k} \text {. }
$$

Thus, making use of (3.8), (3.9) and (3.11),

$$
\begin{aligned}
M_{p}^{p}(f) & =\sum_{n \in Z}|\hat{f}(n)|^{p} \\
& =\sum_{k=0}^{\infty} \sum_{n \in S_{k}}\left|\hat{f}_{k}(n)\right|^{p} \\
& =\sum_{k=0}^{\infty}(k+1)^{-2 p} 2^{-k p / q} 2^{k} \\
& =\sum_{k=0}^{\infty}(k+1)^{-2 p} 2^{k(1-p / q)} \\
& =\infty \text { for } q \in(1,2], p \in[1, q),
\end{aligned}
$$

and so $f \notin \bigcup_{p \in[1, q)} A^{p}$.

We still need to choose $\left(v_{k}\right)$ appropriately. It is sufficient to choose $\left(v_{k}\right)$ to be a strictly monotonic increasing sequence such that

$$
v_{k+1}>v_{k}+2^{k}-1, \quad k=0,1,2, \cdots,
$$

to ensure that the $S_{k}, k=0,1,2, \cdots$, are disjoint. (3.12) is satisfied by the choice

$$
v_{k}=2^{k+1}, \quad k=0,1,2, \cdots,
$$

and our construction is completed.

Construction 3.2. The strict inclusion (3.2).

The method employed here is the same as in 3.1. Similar reasoning shows that, given $q \in[1,2)$,

where

$$
f=\sum_{k=0}^{\infty} f_{k},
$$

$$
f_{k}=(k+1)^{-1 / q} 2^{-k / q} P_{k} e_{2^{k+1}}, \quad k=0,1,2, \cdots,
$$

is such that $f \notin A^{q}$, but $f \in \bigcap_{p \in(q, 2]} A^{p}$.

4. The multipliers $\left(A^{p}, A^{p}\right), p \in[1,2]$

Lemma 4.1. $\left(A^{p}, A^{p}\right)=\left(A^{p}, C\right), p \in[1,2]$.

Proof. Since, for $p \in[1,2], A^{p} \subseteq C$ with a continuous injection, 


$$
\left(A^{p}, A^{p}\right) \subseteq\left(A^{p}, C\right) .
$$

Conversely, suppose $\phi \in\left(A^{p}, C\right), p \in[1,2]$. Then

$$
\|\phi * f\|_{\infty} \leqq \text { const. }\|f\|_{\infty}, \forall f \in T P .
$$

Also, using (2.5),

$$
|\hat{\phi}(n) \cdot \hat{f}(n)| \leqq\|\phi * f\|_{\infty} \leqq \text { const. } N_{p}(f), \forall f \in T P, \forall n \in Z .
$$

Put $f=e_{n}$ in (4.3) to get

$$
|\hat{\phi}(n)| \leqq \text { const., } \forall n \in Z \text {. }
$$

Thus $\phi \in P M$, and so

$$
\|\hat{\phi} \cdot \hat{f}\|_{p} \leqq\|\hat{\phi}\|_{\infty}\|\hat{f}\|_{p}<\infty, \quad \forall f \in A^{p} .
$$

Hence, combination of (4.2) and (4.5) shows that

$$
N_{p}(\phi * f) \leqq \text { const. } N_{p}(f)
$$

and so by (2.4) $\phi \in\left(A^{p}, A^{p}\right)$. Thus

$$
\left(A^{p}, C\right) \subseteq\left(A^{p}, A^{p}\right) .
$$

Combination of (4.1) and (4.6) completes our proof.

4.2 In view of what was said in 2.3 and 2.6 , there is a $1-1$ correspondence $l \leftrightarrow \phi$ between $\left(A^{p}\right)^{\prime}$ and $\left(A^{p}, C\right)$ under which

$$
l(f)=\phi * f(1), \quad \forall f \in T P .
$$

This is equivalent to

$$
l\left(\tau_{x} f\right)=\phi * f(x), \quad \forall f \in T P, \quad \forall x \in T .
$$

LEMMA 4.3. To every $l \in\left(A^{p}\right)^{\prime}$ corresponds $\mu \in M$ and $\sigma \in P M^{p \prime}$ such that

$$
l(f)=\mu * f(1)+\sigma * f(1), \quad \forall f \in T P .
$$

The converse is also true.

Proof. Consider the mapping $f \mapsto(f, \hat{f}), f \in T P$, and define

$$
S=\left\{(f, \hat{f}) \in C \times l^{p}: f \in T P\right\} .
$$

Take $l \in\left(A^{p}\right)^{\prime}$ and define a map $l^{\prime}$ on $S$ by

$$
l^{\prime}:(f, \hat{f}) \mapsto l(f) \text {. }
$$

$l^{\prime}$ is well-defined since

$$
((f, \hat{f})=(g, \hat{g})) \Rightarrow(f=g) .
$$

$l^{\prime}$ is clearly linear; and since 


$$
\left|l^{\prime}((f, \hat{f}))\right|=|l(f)| \leqq \text { const. } N_{p}(f)=\text { const. }\left(\|f\|_{\infty}+\|\hat{f}\|_{p}\right),
$$

$l^{\prime}$ is continuous on $S$ as a subspace of $C \times l^{p}$. Thus, by the Hahn-Banach Theorem, $l^{\prime}$ can be extended to a continuous linear functional on the whole of $C \times l^{p}$. Denote this extension by $l^{\prime}$ also. We can now write

$$
l(f)=l^{\prime}((f, 0))+l^{\prime}((0, \hat{f})), \quad \forall f \in T P .
$$

The mapping $f \mapsto l^{\prime}((f, 0))$ is a continuous linear functional on $C$, so it can be represented by a measure, $\mu \in M$, such that

$$
l^{\prime}((f, 0))=\langle\check{\mu}, f\rangle=\mu * f(1), \quad \forall f \in C .
$$

Also, $\theta \mapsto l^{\prime}((0, \theta))$ is a continuous linear functional on $l^{p}, p \in[1,2]$, so it can be represented by an element, $\alpha \in l^{p^{\prime}}$, such that

$$
l^{\prime}((0, \theta))=\sum_{n \in Z} \alpha(n) \theta(n) .
$$

Define $\sigma \in P M^{p^{\prime}}$ by

$$
\hat{\sigma}(n)=\alpha(n), \quad \forall n \in Z .
$$

Then, for $f \in T P$,

$$
\sigma * f=\sum_{n \in Z} \hat{\sigma}(n) \hat{f}(n) e_{n} .
$$

Thus, by (4.15), we can write

$$
\sigma * f(1)=\sum_{n \in Z} \alpha(n) \hat{f}(n)=l^{\prime}((0, \hat{f})), \quad \forall f \in T P .
$$

Combination of (4.13), (4.14) and (4.18) gives

$$
l(f)=\mu * f(1)+\sigma * f(1), \quad \forall f \in T P,
$$

where $\mu \in M$ and $\sigma \in P M^{p^{\prime}}$.

Conversely, suppose $\mu \in M$ and $\sigma \in P M^{p^{\prime}}$. Consider the map $l: f \mapsto \mu * f(1)$ $+\sigma * f(1)$ on $T P$. We see that, for every $f \in T P$,

$$
\begin{aligned}
|l(f)| \leqq|\mu * f(1)|+|\sigma * f(1)| & \leqq\|\mu * f\|_{\infty}+\|\sigma * f\|_{\infty} \\
& \leqq\|\mu\|\|f\|_{\infty}+\|\hat{\sigma}\|_{p^{\prime}}\|\hat{f}\|_{p} \\
& \leqq \text { const. } N_{p}(f) .
\end{aligned}
$$

Thus $l \in\left(A^{p}\right)^{\prime}$.

THEOREM 4.4. $\left(A^{p}, C\right)=\left(A^{p}, A^{p}\right)=M+P M^{p^{\prime}}, \quad p \in[1,2]$.

Proof. By 4.1, $\left(A^{p}, A^{p}\right)=\left(A^{p}, C\right)$ for $p \in[1,2]$. By $4.2, \phi \in\left(A^{p}, C\right)$ if and only if $\phi$ is such that 


$$
\phi * f(x)=l\left(\tau_{x} f\right), \quad \forall f \in T P, \quad \forall x \in T,
$$

for some $l \in\left(A^{p}\right)^{\prime}$. Thus, by $4.3, \phi \in\left(A^{p}, C\right)$ if and only if there exist $\mu \in M, \sigma \in P M^{p^{\prime}}$ such that, for every $f \in T P$ and ever $y \in T$,

$$
\begin{aligned}
\phi * f(x)=l\left(\tau_{x} f\right) & =\mu * \tau_{x} f(1)+\sigma * \tau_{x} f(1) \\
& =\mu * f(x)+\sigma * f(x)
\end{aligned}
$$

This signifies that $\phi=\mu+\sigma$.

\section{Strict inclusion results for $\left(A^{p}, A^{p}\right), p \in[1,2]$}

Firstly, we will prove the following strict inclusion results:

$$
\left(A^{q}\right)^{\prime} \underset{p}{\mp} \bigcap_{p \in[1, q)}\left(A^{p}\right)^{\prime} \text { if } q \in(1,2]
$$

and

$$
\bigcup_{p \in(q, 2]}\left(A^{p}\right)^{\prime} \underset{\mp}{\mp}\left(A^{q}\right)^{\prime} \text { if } q \in[1,2)
$$

We note here that the wide inclusion " $\subseteq$ " in (5.1) and (5.2) is trivial, since $N_{r}$ is stronger than $N_{s}$ if $r<s$.

Construction 5.1. The strict inclusion (5.1).

Consider a given $q \in(1,2]$. We wish to construct a linear functional, $l$ say, on the space $T P$, such that

$$
l(f)=\sum_{n \in Z} c_{n} \hat{f}(n), \quad f \in T P,
$$

where $\left(c_{n}\right)$ is chosen so that $l$ is not continuous in the topology induced by $A^{q}$, but $l$ is continuous in the topology induced by $\boldsymbol{A}^{p}$, for every $p \in[1, q)$. For $p \in(1, q)$ it is sufficient to choose $\left(c_{n}\right) \in l^{p^{\prime}}$, for then, for every $f \in T P$,

$$
|l(f)| \leqq\left|\sum_{n \in Z} c_{n} \hat{f}(n)\right| \leqq\left\|\left(c_{n}\right)\right\|_{p^{\prime}} M_{p}(f) \leqq\left\|\left(c_{n}\right)\right\|_{p^{\prime}} N_{p}(f)
$$

Now define

$$
f_{k}=\beta_{k, q} P_{k} e_{v_{k}}, \quad k=0,1,2, \cdots,
$$

where $\left(\beta_{k, q}\right)$ and $\left(v_{k}\right)$ will be chosen appropriately, the latter to ensure that the $S_{k}=\operatorname{sp}\left(f_{k}\right)$ are disjoint. We have

$$
\hat{f}_{k}(n)= \begin{cases}\varepsilon_{k}(n) \beta_{k, q} & n \in S_{k} \\ 0 & n \notin S_{k}\end{cases}
$$

Put

$$
c_{n}= \begin{cases}b_{k, q} \operatorname{sgn} \hat{f}_{k}(n)\left|\hat{f}_{k}(n)\right|^{q-1} & n \in S_{k} \\ 0 & n \notin \bigcup_{k} S_{k}\end{cases}
$$


where $\left(b_{k, q}\right)$ is a sequence of positive terms which will be chosen appropriately. Then $\left(c_{n}\right) \in l^{p^{\prime}}$ if and only if

$$
\left\|\left(c_{n}\right)\right\|_{p^{\prime}}^{p^{\prime}}=\sum_{n \in Z}\left|c_{n}\right|^{p^{\prime}}=\sum_{k=0}^{\infty} b_{k, q}^{p^{\prime}} \sum_{n \in S_{k}}\left|\hat{f}_{k}(n)\right|^{(q-1) p^{\prime}}<\infty ;
$$

which is equivalent to

$$
\left\|\left(c_{n}\right)\right\|_{p^{\prime}}^{p^{\prime}}=\sum_{k=0}^{\infty} b_{k, q}^{p^{\prime}} \beta_{k, q}^{(q-1) p^{\prime}}\left|S_{k}\right|<\infty .
$$

To ensure that $l$ is not continuous in the topology induced by $A^{q}$, we seek to arrange that

By (2.1), (2.2) and (5.4),

$$
\sup _{k}\left(\frac{\left|l\left(f_{k}\right)\right|}{N_{q}\left(f_{k}\right)}\right)=\infty
$$

Also,

$$
N_{q}\left(f_{k}\right) \sim \beta_{k . q} 2^{k / q} .
$$

$$
\left|l\left(f_{k}\right)\right|=\left|\sum_{n \in Z} c_{n} \hat{f}_{k}(n)\right|=\sum_{n \in S_{k}} b_{k, q}\left|\hat{f}_{k}(n)\right|^{q}=b_{k, q} \beta_{k, q}^{q}\left|S_{k}\right| .
$$

Thus (5.8) can be replaced by the condition

$$
\sup _{k}\left(\frac{b_{k, q} \beta_{k, q}^{q}\left|S_{k}\right|}{\beta_{k, q} 2^{k / q}}\right)=\infty ;
$$

that is, by

$$
\sup _{k}\left(b_{k, q} \beta_{k, q}^{(q-1)} 2^{-k / q}\left|S_{k}\right|\right)=\infty
$$

(3.10) and (3.11) apply here, so (5.9) becomes

$$
\sup _{k}\left(b_{k, q} \beta_{k, q}^{(q-1)} 2^{-k / q} 2^{k}\right)=\infty
$$

Choose

$$
b_{k, q}=(k+1)^{-1 / q^{\prime}} ; \beta_{k, q}=(k+1)^{2 / q} 2^{-k / q}, \quad k=0,1,2, \cdots .
$$

Then (5.7) is satisfied, since

$$
\begin{aligned}
\left\|\left(c_{n}\right)\right\|_{p^{\prime}}^{p^{\prime}} & =\sum_{k=0}^{\infty}(k+1)^{-p^{\prime} / q^{\prime}}(k+1)^{2\left(q^{-1}\right) p^{\prime} / q} 2^{-k(q-1) p^{\prime} / q} 2^{k} \\
& =\sum_{k=0}^{\infty}(k+1)^{p^{\prime} / q^{\prime}} 2^{-k\left(p^{\prime} / q^{\prime}-1\right)} \\
& <\infty \quad \text { for } p \in(1, q) .
\end{aligned}
$$

Also, (5.9) is satisfied, since 


$$
\begin{aligned}
& \sup _{k}\left((k+1)^{-1 / q^{\prime}}(k+1)^{2(q-1) / q} 2^{-k(q-1) / q} 2^{-k / q} 2^{k}\right) \\
& =\sup _{k}\left[(k+1)^{1 / q^{\prime}}\right]=\infty \quad \text { for } q \in(1,2] .
\end{aligned}
$$

As in 3.1 we can choose $\left(v_{k}\right)$ such that

$$
v_{k}=2^{k+1}, \quad k=0,1,2, \cdots .
$$

For the case $q \in(1,2]$ and $p=1$, it is sufficient to have $\left(c_{n}\right) \in l^{\infty}$. From (5.6) and (5.11),

$$
\left\|\left(c_{n}\right)\right\|_{\infty}=\sup _{n \in Z}\left|c_{n}\right|=\sup _{k}\left((k+1)^{1 / q^{\prime}} 2^{-k / q^{\prime}}\right) .
$$

Since

$$
(k+1)^{1 / q^{\prime}} 2^{-k / q^{\prime}} \leqq 1, \quad \forall k \geqq 0, \quad q \in(1,2],
$$

we see that $\left\|\left(c_{n}\right)\right\|_{\infty}=1<\infty$, and so $\left(c_{n}\right) \in l^{\infty}$, and our construction is completed.

Construction 5.2. The strict inclusion (5.2).

Consider a given $q \in(1,2)$. The method employed here is the same as in 5.1, and similar reasoning shows that the linear functional, $l$, on $T P$, defined by

$$
l(f)=\sum_{n \in Z} c_{n} \hat{f}(n)
$$

where

$$
c_{n}= \begin{cases}b_{k, q} \operatorname{sgn} \hat{f}_{k}(n)\left|\hat{f}_{k}(n)\right| & n \in S_{k} \\ 0 & n \notin \bigcup_{k} S_{k},\end{cases}
$$

is such that $l$ is continuous in the topology induced by $A^{q}, q \in(1,2)$, but $l$ is not continuous in the topology induced by $A^{p}$ for every $p \in(q, 2]$.

We now consider the case $q=1$. We want to construct a suitable linear functional, $l$, on $T P$, of the form given in (5.13). $\left(c_{n}\right) \in l^{\infty}$ is a sufficient condition for $l$ to be continuous in the topology induced by $A^{1}=A$. Choose

$$
c_{n}= \begin{cases}b_{k} \operatorname{sgn} \hat{f}_{k}(n) & n \in S_{k} \\ 0 & n \notin \bigcup_{k} S_{k},\end{cases}
$$

where $\left(b_{k}\right)$ is a sequence of positive terms which we will choose appropriately, and

$$
f_{k}=P_{k} e_{v_{k}}, \quad k=0,1,2, \cdots
$$


To ensure that $l$ is not continuous in the topology induced by $A^{p}, p \in(1,2]$, we seek to arrange that

$$
\sup _{k}\left(\frac{\left|l\left(f_{k}\right)\right|}{N_{p}\left(f_{k}\right)}\right)=\infty, \quad \forall p \in(1,2]
$$

By (2.1), (2.2) and (5.18),

and

$$
N_{p}\left(f_{k}\right) \sim 2^{k / p}
$$

$$
\left|l\left(f_{k}\right)\right|=\left|\sum_{n \in Z} c_{n} \hat{f}_{k}(n)\right|=\sum_{n \in S_{k}} b_{k}\left|\hat{f}_{k}(n)\right|=b_{k}\left|S_{k}\right|,
$$

so we can replace (5.19) by

$$
\sup _{k}\left(b_{k}\left|S_{k}\right| 2^{-k / p}\right)=\infty, \quad \forall p \in(1,2] .
$$

(3.10) and (3.11) apply here, so (5.20) becomes

$$
\sup _{k}\left[b_{k} 2^{k(1-1 / p)}\right]=\infty, \quad \forall p \in(1,2] .
$$

Choose

$$
b_{k}=1, k=0,1,2, \cdots \text {. }
$$

Then $\left(c_{n}\right) \in l^{\infty}$ since

$$
\left\|\left(c_{n}\right)\right\|_{\infty}=\sup _{n \in Z}\left|c_{n}\right|=1<\infty ;
$$

and (5.21) is satisfied since

$$
\sup _{k}\left(2^{k(1-1 / p)}\right)=\infty, \quad \forall p \in(1,2] .
$$

Again, as in 3.1, we can choose $\left(v_{k}\right)$ such that

$$
v_{k}=2^{k+1}, k=0,1,2, \cdots,
$$

and our construction is completed.

THEOREM 5.3. The following strict inclusions hold:

and

$$
\left(A^{q}, A^{q}\right) \underset{p \in[1, q)}{\subsetneq}\left(A^{p}, A^{p}\right) \text { if } q \in(1,2],
$$

$$
\bigcup_{p \in(q, 2]}\left(A^{p}, A^{p}\right) \underset{f}{\subsetneq}\left(A^{q}, A^{q}\right) \text { if } q \in[1,2)
$$

Proof. By 5.1, if $q \in(1,2]$, then $\exists l \in \bigcap_{p \in[1, q)}\left(A^{p}\right)^{\prime}, l \notin\left(A^{q}\right)^{\prime}$. Let $\phi$ correspond to $l$ as in 4.2. Then $\phi \in\left(A^{p}, C\right), \forall p \in[1, q)$ and $\phi \notin\left(A^{q}, C\right)$. Use of 4.1 gives the result (5.23). 
Similar resoning can be used to derive (5.24) from 5.2.

6. The multipliers $\left(A^{p}, A^{q}\right), p \in[1,2], q \in[p, 2]$

THEOREM 6.1. $\left(A^{p}, A^{q}\right)=\left(A^{p}, A^{p}\right)=M+P M^{p^{\prime}}, p \in[1,2], q \in[p, 2]$.

Proof. For $q \in[p, 2], A^{p} \subseteq A^{q}$ with continuous injection, so

$$
\left(A^{p}, A^{q}\right) \supseteq\left(A^{p}, A^{p}\right), p \in[1,2], q \in[p, 2] .
$$

Conversely, since $A^{q} \subseteq C$ with continuous injection,

$$
\left(A^{p}, A^{q}\right) \subseteq\left(A^{p}, C\right), p \in[1,2], q \in[p, 2] .
$$

Use of 4.1 with (6.2) gives

$$
\left(A^{p}, A^{q}\right) \subseteq\left(A^{p}, A^{p}\right), p \in[1,2], q \in[p, 2] .
$$

Combine (6.1) and (6.3) and then use 4.4 to deduce the required result.

7. The multipliers $\left(A^{p}, A^{q}\right), p \in[1,2], q \in[1, p)$.

THEOREM 7.1. $M^{p q /(p-q)} \subseteq\left(A^{p}, A^{q}\right), p \in[1,2], q \in[1, p)$.

Proof. Consider $\mu \in M^{p q /(p-q)}$. Then, since $\mu \in M$,

$$
\|\mu * f\|_{\infty} \leqq\|\mu\|\|f\|_{\infty}, \forall f \in T P, p \in[1,2] .
$$

Also, Hölder's inequality gives for every $f \in T P$

$$
\sum_{n \in Z}|\hat{\mu}(n) \hat{f}(n)|^{q} \leqq\left(\sum_{n \in Z}|\hat{f}(n)|^{q s}\right)^{1 / s}\left(\sum_{n \in Z}|\hat{\mu}(n)|^{q s^{\prime}}\right)^{1 / s^{\prime}} .
$$

For $s=p / q, s^{\prime}=p /(p-q)$, this becomes

(7.2) $\sum_{n \in Z}|\hat{\mu}(n) \hat{f}(n)|^{q} \leqq\left(\sum_{n \in Z}|\hat{f}(n)|^{p}\right)^{q / p} \leqq\left(\sum_{n \in Z}|\hat{\mu}(n)|^{p q /(p-q)}\right)^{(p-q) / p q}, \quad \forall f \in T P$.

By (7.1) and (7.2), we have for $f \in T P$

$$
\begin{aligned}
N_{q}(\mu * f) & =\|\mu * f\|_{\infty}+\|\widehat{\mu * f}\|_{q} \\
& \leqq\|\mu\|\|f\|_{\infty}+\|\hat{\mu}\|_{p q /(p-q)}\|\hat{f}\|_{p} \leqq \text { const. } N_{p}(f) .
\end{aligned}
$$

Now refer to (2.4).

THEOREM 7.2. $\phi \in\left(A^{2}, A^{q}\right) \Rightarrow \phi \in l^{2 q /(2-q)}, q \in[1,2)$.

Proof. From [3], Corollary 2.3, p. 468 it follows that, if $\hat{\phi} \cdot \hat{f} \in l^{q}(Z), q \in[1,2)$, for each $f \in C(T)$, then $\hat{\phi} \in l^{2 q /(2-q)}$. Since $A^{2}=C$, our result follows directly.

THEOREM 7.3. $(C, A)=L^{2}$. 
Proof. From 7.1,

$$
L^{2}=M^{2} \subseteq\left(A^{2}, A^{1}\right)=(C, A) .
$$

Conversely, suppose $\phi \in(C, A)$. Then, by $7.2, \hat{\phi} \in l^{2}$, and so $\phi \in L^{2}$. Thus $(C, A) \subseteq L^{2}$.

7.4 We now establish preliminary results leading to a necessary condition for $\phi \in\left(A^{p}, A^{q}\right), p \in[1,2], q \in[1, p)$.

Consider

$$
S=\left\{\left(c_{n}\right) \in l^{p}: \sum_{n \in Z \backslash\{0\}}\left|c_{n}\right|^{2} \log ^{1+\varepsilon}|n|<\infty, \varepsilon>0\right\} .
$$

Then, from [2], 14.3.6, p. 205, for $\left(c_{n}\right) \in S$, almost all the series

$$
\sum_{n \in Z} r_{|n|}(t) c_{n} e_{n}
$$

are the Fourier series of continuous functions. (In fact, of functions in $A^{p}$ ). If $\phi \in\left(A^{p}, A^{q}\right), p \in[1,2], p \in[1,2], q \in[1, p)$, then

and so

$$
\phi * f \in A^{q}, \forall f \in A^{p},
$$

$$
\left(\sum_{n \in Z}\left|\hat{\phi}(n) c_{n}\right|^{q}\right)^{1 / q}<\infty, \forall c=\left(c_{n}\right) \in S .
$$

Define a map $Q_{\phi}: S \rightarrow l^{q}$ by

$$
Q_{\phi}:\left(c_{n}\right) \mapsto\left(\hat{\phi}(n) c_{n}\right) .
$$

$Q_{\phi}$ is clearly linear. It is not hard to see that $S$ is a Banach space under the norm

$$
\|\cdot\|_{S}:\left(c_{n}\right) \mapsto\left(\sum_{n \in Z}\left|c_{n}\right|^{p}\right)^{1 / p}+\left(\sum_{n \in Z \backslash\{0\}}\left|c_{n}\right|^{2} \log ^{1+\varepsilon}|n|\right)^{1 / 2} .
$$

An application of the Closed Graph Theorem shows that $Q_{\phi}$ is a continuous map from $S$ to $l^{q}$, so we have

$$
\left(\sum_{n \in Z}\left|\hat{\phi}(n) c_{n}\right|^{q}\right)^{1 / q} \leqq K\left[\left(\sum_{n \in Z}\left|c_{n}\right|^{p}\right)^{1 / p}+\left(\sum_{n \in Z \backslash\{0\}}\left|c_{n}\right|^{2} \log ^{1+\varepsilon}|n|\right)^{1 / 2}\right],
$$

where $K=K(\phi, \varepsilon)$ is a constant.

THeOREM 7.5. If $\phi \in\left(A^{p}, A^{q}\right), p \in[1,2], q \in[1, p)$, then for every $\varepsilon>0$,

$$
\sum_{|n| \leqq R}|\hat{\phi}(n)|^{p q /(p-q)} \leqq \max \left[1,\left(K\left(1+J_{R}\right)\right)^{p q /(p-q)}\right],
$$

where $K=K(\phi, \varepsilon)$ is independent of $R$ and

$$
J_{R}=\max _{0<|n| \leqq R}\left(|\hat{\phi}(n)|^{(2-p) q /(p-q)} \log ^{1+\varepsilon}|n|\right) .
$$


Proof. Suppose $\phi \in\left(A^{p}, A^{q}\right), p \in[1,2], q \in[1, p)$. Then (7.5) holds. Choose $\left(c_{n}\right) \in S$ such that

$$
c_{n}= \begin{cases}|\hat{\phi}(n)|^{q /(p-q)} & |n| \leqq R \\ 0 & |n|>R,\end{cases}
$$

and consider $J_{R}$ as defined in (7.6). Then, on writing $\sigma_{R}=\Sigma_{|n| \leqq R}|\hat{\phi}(n)|^{p q /(p-q)}$, (7.5) yields

$$
\sigma_{R}^{1 / q} \leqq K\left(\sigma_{R}^{1 / p}+J_{R} \sigma_{R}^{1 / 2}\right)
$$

that is,

$$
\sigma_{R}^{1 / q-1 / p} \leqq K\left(1+J_{R} \sigma_{R}^{1 / 2-1 / p}\right)
$$

It follows that

$$
\sigma_{R} \leqq \max \left[1,\left(K\left(1+J_{R}\right)\right)^{p q /(p-q)}\right] .
$$

Corollary 7.6. If $\phi \in\left(A^{p}, A^{q}\right), p \in[1,2], q \in[1, p)$, then

$$
\sum_{|n| \leqq R}|\hat{\phi}(n)|^{p q /(p-q)}=O\left\{(\log R)^{\Delta}\right\}
$$

where $\Delta=(1+\varepsilon) p q / 2(p-q)$, and $\varepsilon>0$.

Proof. Suppose $\phi \in\left(A^{p}, A^{q}\right), p \in[1,2], q \in[1, p)$. Then $\phi \in(A, C)=P M$, and it follows that $J_{R}=O\left\{(\log R)^{(1+\varepsilon) / 2}\right\}$, where $J_{R}$ is defined in (7.6). Application of 7.5 now gives the desired result.

CoRollary 7.7. If $\phi \in\left(A^{p}, A^{q}\right), p \in[1,2], q \in[1, p)$, and $J_{R}=O(1)$, where $J_{R}$ is defined in (7.6), then $\hat{\phi} \in l^{p q /(p-q)}$.

Proof. This result follows directly from 7.5.

\section{References}

[1] Lynette M. Butler, 'Certain non-algebras in harmonic analysis', Bull. Austral. Math. Soc. 4 (1971), 247-254.

[2] R. E. Edwards, Fourier Series: A Modern Introduction. Vol. II. (Holt, Rinehart and Winston, Inc. 1967).

[3] R. E. Edwards, 'Changing signs of Fourier coefficients', Pacific J. Math. 15 (1965), 463-475.

[4] Y. Katznelson, An Introduction to Harmonic Analysis (John Wiley, 1968).

School of General Studies

Australian National University

Canberra A.C.T. 2600 